\title{
Épouser un gendarme ou épouser la gendarmerie? Les femmes de gendarmes entre contrôle matrimonial et contrôle social
}

Marc Bergère

\section{(2) OpenEdition}

Journals

Édition électronique

URL : https://journals.openedition.org/clio/1411

DOI : 10.4000/clio.1411

ISSN : $1777-5299$

Éditeur

Belin

\section{Édition imprimée}

Date de publication : 1 novembre 2004

Pagination : 123-134

ISBN : 2-85816-755-9

ISSN : $1252-7017$

\section{Référence électronique}

Marc Bergère, «Épouser un gendarme ou épouser la gendarmerie ? Les femmes de gendarmes entre contrôle matrimonial et contrôle social », Clio. Histoire, femmes et sociétés [En ligne], 20 | 2004, mis en ligne le 01 janvier 2007, consulté le 22 avril 2022. URL : http://journals.openedition.org/clio/1411 ; DOI : https://doi.org/10.4000/clio.1411

Ce document a été généré automatiquement le 22 avril 2022.

Tous droits réservés 


\title{
Épouser un gendarme ou épouser la gendarmerie? Les femmes de gendarmes entre contrôle matrimonial et contrôle social
}

\author{
Marc Bergère
}

1 Le 11 août 1989, alors que la gendarmerie est secouée depuis plusieurs semaines par une véritable «mutinerie verbale $»^{1}$, Jean-Michel Boucheron, président socialiste de la commission Défense de l'Assemblée nationale, déclare :

Il est bien évident qu'aujourd'hui tout ne va pas bien. Je pense notamment aux femmes de gendarmes qui doivent compter avec un mari qui n'est jamais sûr de pouvoir prendre ses week-ends, qui voit toutes ses soirées menacées par un coup de téléphone. Par ailleurs, l'atmosphère relativement confinée de la vie en caserne donne aux femmes de gendarmes l'impression de ne pas vivre comme les autres femmes françaises. Il nous faut aussi prendre cela en considération. ${ }^{2}$

2 Les gendarmes ont souvent coutume de dire lorsqu'une femme épouse l'un des leurs qu'elle épouse aussi la gendarmerie: dès lors il n'est pas rare que l'institution soit présentée, et aussi parfois perçue par les intéressées, comme "une rivale $»^{3}$ ou encore " une maitresse exigeante $~^{4}$. Au-delà du cliché, l'image n'en recouvre pas moins une part de réalité des rapports de genre au sein d'une institution traditionnellement masculine. Appuyé sur les textes normatifs, relatifs au mariage et à la réglementation de la vie privée des gendarmes, cet article témoigne du contrôle matrimonial et social imposé aux femmes de gendarmes en raison du statut professionnel de leur mari.

Entre contrôle matrimonial...

3 À compter de 1808, la gendarmerie à l'instar des autres corps d'armées exerce un contrôle sur les futures épouses de ses hommes. Afin d'empêcher «les militaires de contracter des mariages inconvenants susceptibles d'altérer la considération due à leur caractère $»^{5}$, tout projet de mariage est désormais soumis à une autorisation de la hiérarchie. Cette prescription, inscrite au règlement sur le service intérieur de la gendarmerie de $1933^{6}$, reste en vigueur jusqu'à la fin des années 1970. L'autorisation 
préalable s'accompagnait d'une enquête de voisinage effectuée, en général, par la brigade de résidence de la future épouse et transmise ensuite par la voie hiérarchique. Enquête de moralité et d'honorabilité, la procédure s'est doublée jusqu'au lendemain de la Seconde Guerre mondiale d'une enquête relative à la fortune de «la prétendante »: bien que l'obligation de la dot ait disparu en 1900, le capital apparait encore dans l'entre-deux-guerres comme un gage déterminant d'honorabilité. Si l'on excepte cette disposition tombée ensuite en désuétude, force est de constater que les conditions requises et les qualités attendues pour contracter un mariage ont peu varié au $\mathrm{XX}^{\mathrm{e}}$ siècle. En règle générale, l'enquête intégrait non seulement la situation présente de la future épouse (résidence, profession...) mais également ses antécédents (éducation, formation, conduite, mode d'existence...). Elle s'étendait aussi à la situation et réputation de ses parents, et de ses frères et sœurs. À partir de 1918, les épouses fonctionnaires ou employées titulaires d'une administration publique ont bénéficié de clauses particulières : il n'était pas effectué d'enquête de moralité les concernant et le certificat de bonne vie et mœurs était remplacé par un certificat d'exercice délivré par leur chef de service ${ }^{7}$. A contrario, le régime de Vichy a adopté des mesures plus coercitives : « en principe toute autorisation de contracter mariage avec une personne de nationalité étrangère ou de race juive sera refusée aux personnels de la gendarmerie [...] »; et l'officier « devra prendre l'engagement écrit qu'à dater de son mariage, sa femme cessera tout travail rémunéré $»^{8}$.

4 Au-delà de ces dispositions d'exception marquées par leur époque et la nature profonde du régime concerné, la lecture des rapports d'enquête ${ }^{9}$ démontre que le profil type de la future épouse a peu évolué des années 30 aux années 60. Il importe surtout pour l'intéressée de ne pas avoir trop fait parler d'elle. Respectueuse des valeurs familiales, elle doit avoir un passé "sans tâche $»^{10}$ morale ou politique. Vivre chez ses parents (ou a minima en bonnes relations avec eux) jusqu'au mariage, ne pas trop sortir, ni «être légère ", sont des gages d'honorabilité. La fréquentation de milieux politiques « subversifs» (communistes dans les années 30, collaborateurs au sortir de la guerre...) est particulièrement surveillée. Probité, discrétion, capacité à tenir son ménage (" propre », « courageuse », « travailleuse », « économe » ${ }^{11} .$. ) semblent les qualités les plus recherchées: «elle a reçu de sa mère les principes d'ordre, d'économie et de propreté qui sont l'apanage de la ménagère modèle. $»^{12}$ Ces caractéristiques dessinent le portrait d'une épouse idéale qui se doit surtout «d'être digne d'intégrer la gendarmerie $»^{13}$ et, si possible, femme au foyer. En 1975, dans ses réflexions sur les femmes de gendarmes, le colonel Boyé ne dit pas autre chose :

Tout en restant femme, l'épouse a le sentiment d'être elle aussi un peu gendarme et de partager avec son mari la responsabilité de maintenir élevé le prestige de l'Arme. [...]. Dans leur immense majorité, ces femmes sont heureuses. Elles sont fières de leurs maris, de la haute conception qu'ils ont de leur métier [...]. Elles ont, ellesmêmes, une pleine conscience de l'enrichissement personnel que leur apporte l'effort de dépassement exigé par une vie digne et exemplaire. ${ }^{14}$

5 L'enquête considère également son état de santé, les instructions de 1957 rappelant : « s'il est notoire que la future est atteinte d'une maladie grave et contagieuse, mention en est faite dans le rapport d'enquête afin de permettre au commandement de réserver éventuellement sa décision quant à l'autorisation de loger à la caserne $»^{15}$.

6 Ces restrictions apportées au libre choix du conjoint sont restées en vigueur au sein des armées jusqu'à la loi du 13 juillet 1972, qui abolit l'autorisation préalable pour tous les militaires sauf... les gendarmes, qui vont patienter jusqu'en $1978^{16}$. La justification 
donnée à l'époque à cette exception fut la suivante: "Les gendarmes peuvent être officiers de police judiciaire et, à cause de cette particularité, on tient à ce que leur honorabilité ne puisse être suspectée. On entoure donc leur mariage d'un minimum de précautions qui n'ont rien d'abusif. ${ }^{17}$ Visiblement, une partie des cadres adhéraient à ce point de vue, si l'on en juge par cet extrait de la presse corporatiste :

La femme du gendarme a conscience que sa vie privée doit être irréprochable. Avant le mariage, elle a d'ailleurs fait l'objet d'une enquête de moralité. Cette obligation, supprimée depuis peu pour les mariages de militaires, a été maintenue pour les gendarmes. Il s'agit là d'une mesure de sagesse, dont on comprend aisément les raisons. ${ }^{18}$

7 Depuis 1978, les gendarmes se marient librement. Ils sont cependant tenus de rendre compte de la situation de leur conjoint à leur hiérarchie, qui exerce donc toujours un contrôle, désormais a posteriori. Celui-ci peut encore avoir des incidences, en terme d'affectation ou de mutation dans l'intérêt du service, si l'institution estime que la situation du conjoint ou de son entourage est de nature à compromettre l'indépendance de l'intéressé. De plus, la libéralisation propre au mariage n'a pas tout réglé, comme en témoignent ces réticences exprimées dans la presse professionnelle envers l'union libre dans les années 80 :

Alors que disparaît une formalité administrative destinée à faciliter le mariage, un nouveau type de couple se manifeste décidé à s'en passer. Le mariage à l'essai, comme l'appellent certains, indispose les familles des petites résidences. Le séjour de ces faux-ménages apparaît scandaleux, en contradiction avec la morale et le règlement sur le service intérieur qui autorise seulement les femmes de militaires à loger à la caserne. ${ }^{19}$

... et contrôle social

8 En devenant, durant les mouvements de $1989^{20}$ et de $2000-2001^{21}$, les porte-parole de leurs maris condamnés au silence par leur statut militaire, les épouses de gendarmes ont poussé à son terme une logique qui vise, au sens propre comme au figuré, à les faire " entrer en gendarmerie ", au prix d'un brouillage permanent des sphères privée et publique, intime et professionnelle. Chemin faisant, elles n'en ont pas moins exprimé leur propre malaise face à des contraintes vécues comme de plus en plus pesantes. Au premier rang d'entre elles, arrive l'enfermement de la vie en caserne. Si beaucoup reconnaissent que l'encasernement du gendarme et de sa famille offre certains avantages, tels que la gratuité du logement ou la sécurité, nombreuses sont celles qui souffrent d'un manque d'intimité. De fait, une caserne, surtout dans les petites unités, impose une vie communautaire digne d'un phalanstère ${ }^{22}$. Au cœur de relations non choisies, qui parfois reproduisent l'organisation et les tensions hiérarchiques, il est très difficile de se sentir vraiment chez soi ${ }^{23}$ :

On préférerait gagner un peu plus et habiter en dehors de la caserne. Nous n'avons aucune intimité. Il n'existe pas de boîte à lettres personnalisée pour le courrier. C'est le planton de service qui le relève. Et puis ce portail toujours fermé, on a l'impression d'être en prison. [...] La famille et les amis sont un peu freinés par ça. Idem pour le téléphone, ça passe obligatoirement par le standard. ${ }^{24}$

Femmes de gendarmes : même en amour notre vie est impossible... Sermonnés par le commandant de compagnie parce que mon mari et moi avions fait trop de bruit au lit. ${ }^{25}$

9 Même si ces récriminations ont pu sembler excessives à certains, elles n'ont certainement pas été une surprise pour la hiérarchie. Dès 1975, le colonel Boyé écrivait : 
La vie professionnelle du gendarme constitue une gêne pour sa vie familiale. C'est indiscutable. [...] La vie qu'elles [les épouses de gendarmes] mènent présente des contraintes : exigences morales résultant de la fonction du mari [...], restrictions à la liberté, conséquences de la vie en caserne dans un logement imposé, impossibilité de prévisions [...], solitude des longues attentes [...]. Ces contraintes peuvent paraître pesantes pour de frêles épaules. ${ }^{26}$

10 À la promiscuité et à la pression exercée par le contrôle de ses faits et gestes en interne, et parfois à l'extérieur en raison de l'exemplarité attendue de la femme du gendarme ${ }^{27}$, s'ajoute souvent un sentiment d'isolement. Ce dernier est renforcé par les difficultés rencontrées par les épouses pour exercer un travail salarié du fait de la disponibilité et des fréquentes mutations de leur époux. Comme souvent dans les rapports genre/ travail, la carrière du mari passe en premier. Et la structure familiale, par le réconfort moral ${ }^{28}$ apporté, reste encore souvent perçue par les personnels masculins comme une condition de réussite et d'investissement dans leur métier. Ainsi que l'observe Emmanuel Reynaud ${ }^{29}$, "être célibataire est considéré pour un homme comme une situation particulièrement difficile dans une brigade territoriale [...], car sans épouse, il est obligé de prendre en charge lui-même tous les aspects des tâches ménagères. Il est d'ailleurs courant qu'un gendarme masculin célibataire soit régulièrement invité ou prenne même directement pension chez un collègue masculin marié. »

11 A contrario, la réciproque n'est pas vraie, la carrière des femmes dans la gendarmerie ${ }^{30}$ apparaissant souvent entravée par l'activité du conjoint et la prise en charge de la maison et des enfants. Pour François Dieu, la conciliation problématique entre les exigences du métier de gendarme et la vie de famille participe pour une large part au taux élevé de sous-officiers féminins qui quittent prématurément la gendarmerie (taux de démission volontaire de 19\% parmi les femmes recrutées ente 1984 et 1995) ${ }^{31}$. Ainsi le célibat, en général assez mal vécu par les personnels masculins, demeure en revanche l'un des plus efficaces facteurs de promotion pour les personnels féminins. Des entretiens réalisés par Emmanuel Reynaud avec 16 gendarmes (10 femmes, 4 hommes et 1 couple $)^{32}$, il ressort que cette inégalité fondamentale est surtout le reflet de représentations masculines de la répartition sexuée des tâches au sein du couple. Ainsi un gendarme masculin assure-t-il d'une collègue : « Tant qu'elle est célibataire, il n'y a pas de problème. Le jour qu'elle va se marier... La popote par exemple, faudra bien que ça soit elle qui la fasse." Un autre affirme à propos d'une collègue mariée : " J'estime même qu'elle est doublement courageuse, parce que c'est pas marrant avec la vie qu'on mène nous au vu du nombre d'heures qu'on a. Elle a son travail de femme à faire en rentrant... Même qu'il y ait pas d'enfants, il faut quand même faire à manger, il faut quand même faire son ménage. Il y a pas... il y a pas à tortiller, hein. Alors que nous, on rentre, il y a toujours la femme qui fait quelque chose, qui fait le manger ou n'importe quoi : on n'a pas ces problèmes là. » Il convient de souligner que l'adaptation des femmes-gendarmes est souvent compliquée par des relations difficiles avec leurs collègues masculins, chez qui l'association gendarmerie / "métier d'homme » reste répandu. Paradoxalement, les relations ne sont guère plus faciles avec les épouses de ces mêmes collègues, qui supportent parfois mal la proximité professionnelle et le temps partagé avec leur époux, particulièrement à l'échelle des brigades territoriales, où les femmes gendarmes sont massivement affectées.

Bon nombre d'épouses de gendarmes sont aussi condamnées à rester des citoyennes inachevées ou inaccomplies en raison «de l'impossibilité d'exercer une profession ou une activité syndicale et politique susceptible de porter atteinte à l'indépendance du 
mari. $»^{33}$ La continuité de la condition militaire conduit enfin le gendarme à le demeurer dans sa vie privée. Le guide du gendarme ${ }^{34}$ l'affirme, il est responsable disciplinairement du comportement des siens. À ce sujet, dans une édition antérieure aux années 1970, on pouvait lire :

Votre vie familiale, pour être ordonnée et correcte, exige une discipline dont vous êtes le principal artisan et le soutien constant, vous devez donc être conscient de votre responsabilité de chef de famille. En effet, comme un simple citoyen mais à plus forte raison à titre militaire, vous êtes seul responsable de votre famille: épouse et enfants. Ce principe implique donc que vous seul serez mis en cause disciplinairement et pécuniairement pour une faute d'un membre de votre famille. Sans vouloir s'immiscer dans vos affaires personnelles, le commandement se réserve la possibilité d'intervenir lorsqu'il y a atteinte à la considération de l'Arme : mauvaise conduite notoire, scandale, dettes, etc. ${ }^{35}$

$13 \mathrm{Si}$, depuis cette date, le ton a changé, la philosophie est restée la même quant au nécessaire contrôle du couple et de la famille. Récemment revu et corrigé, Le guide $d u$ gendarme souligne toujours les devoirs imposés par le port de l'uniforme: «Faites attention à vos fréquentations, n'ignorez pas celles de votre conjoint et de vos enfants qui vous engageront inexorablement [...]. La liberté de votre vie privée ne saurait vous conduire à des attitudes, une tenue ou des propos susceptibles d'altérer la crédibilité de votre efficacité en service et/ou l'image de la gendarmerie. $»^{36}$

La dimension totale de la fonction "gendarmique » tend à effacer ou gommer la séparation entre vie et espace professionnels et privés. Chemin faisant, les épouses sont, bon gré mal gré, partie intégrante de la communauté professionnelle de leur mari. La juxtaposition de ces sphères, qui ne cessent de se croiser au point parfois de se confondre, conduit à une forme "d'intimité collective ${ }^{37}$ propre à cette population. Pendant une grande partie du siècle, ce mode d'organisation a été profondément enraciné dans la culture du corps, participant de son identité voire d'un certain «modèle». Nul doute cependant que les contestations de 1989 et 2001, au sein desquelles la question des femmes a pesé d'un poids non négligeable, la multiplication des situations de célibat géographique mais aussi la menace des divorces ${ }^{38}$ ouvrent désormais, dans un contexte d'évolution globale de la société, sur une quête de modernité y compris au regard de nouveaux rapports de genre.

\section{BIBLIOGRAPHIE}

Boye (Colonel), 1975, «Quelques réflexions sur les femmes de gendarmes », Revue d'études et

d'informations de la gendarmerie nationale, $\mathrm{n}^{\circ} 106, \mathrm{pp}$. 5-10.

CARTAYRade Cyril, 2003, « Le marié était en bleu. Les mariages de gendarmes dans le Puy-de-Dôme au XIX siècle », Sociétés et Représentations, $\mathrm{n}^{\circ}$ 16, spécial « Figures de gendarmes » sous la dir. de Jean-Noël Luc, pp. 269-279.

CLEMENT Sylvie, 2003, Vivre en caserne à l'aube du XXI siècle. L'exemple de la gendarmerie, Paris, L'Harmattan. 
DIEU François, 1999, « Autopsie d'un mouvement social atypique : la fronde des gendarmes de l'été 1989. Dix ans après ", Revue internationale de criminologie et de police technique et scientifique, 4/99, pp. 414-429.

-, 2002, La Gendarmerie, secrets d'un corps, Bruxelles, Complexe.

LAMBERT ( chef d'escadron ), 1984, « Le mariage dans les armées, la maréchaussée et la gendarmerie ", Revue d'études et d'informations de la gendarmerie nationale, n 139 et 140, pp. 54-62 et $49-53$.

REYNAUD Emmanuel, 1988, Les femmes, la violence et l'armée : essai sur la féminisation des armées, Paris, Fondation pour les études de défense nationale.

\section{NOTES}

1. Durant l'été 1989 , la gendarmerie nationale connaît un mouvement de contestation sans précédent, qui se manifeste surtout par l'envoi à la hiérarchie et à la presse de multiples lettres ou pétitions, en général anonymes.

2. Interview accordée au journal Libération, le 11 août 1989, p. 19.

3. Cette notion de rivale revient à plusieurs reprises dans une enquête, interne et informelle, réalisée en 1975 auprès de 76 femmes de gendarmes, Boyé 1975.

4. Dieu $2002: 208$.

5. Selon les termes du Conseil d'État, au sujet du décret du 16 juin 1808, Lambert 1984 : 59.

6. Article 105 du décret du 17 juillet 1933.

7. Modification au tableau des modèles annexé au règlement du 3 février 1914 sur le service intérieur de la gendarmerie départementale (bordereau énumératif des pièces jointes à la demande en mariage), le 8 octobre 1918. SHGN, Mémorial de la gendarmerie, 18ad.37, p. 545 (recueil des textes officiels ou réglementaires relatifs à la gendarmerie, collection reliée par année disponible au Service Historique de la Gendarmerie Nationale, SHGN, Fort de Charenton, Maisons-Alfort). À noter, qu'à l'exception des officiers, tous les gendarmes appartiennent au corps des sous-officiers de gendarmerie, en vertu du décret du 21 février 1918, « donnant aux gendarmes le rang de sousofficiers », SHGN, Mémorial de la gendarmerie, 18ad.37, p. 212.

8. Instruction $n^{\circ} 27296.1 /$ gend du 6 octobre 1942 relative au mariage des militaires de la gendarmerie. Article III : dispositions spéciales, SHGN, Mémorial de la gendarmerie, 18ad.61, p. 329-335. Le dernier point renoue avec une tradition en vogue au XIX ${ }^{e}$ siècle, selon laquelle : «L'officier ne peut compter sur les revenus professionnels de son épouse. La femme de l'officier ne doit pas travailler pour gagner sa vie : c'est là un postulat tenace et virulent. Elle doit rester une maitresse de maison disponible qui se borne à recevoir avec grâce, à donner des ordres à l'ordonnance ou aux domestiques et à surveiller l'éducation des jeunes enfants. Les seules professions acceptables, à la rigueur, sont celles d'institutrice ou de professeur de piano. " (Lambert 1984: 61). 9. Consignés dans les registres de correspondance confidentielle ou courante (R4/R2) aux différents échelons de la hiérarchie (brigade, section, compagnie...).

10. «Mademoiselle $C$. recherchée en mariage par le gendarme H. est de nationalité française à titre originaire, elle n'est pas de race juive. [...] Elle n'a jamais quitté ses parents, sa réputation est sans tâche », SHGN, 52 E 79, BT Bourbonne-les-Bains, 46/4, 22 novembre 1943. 
11. Mentions courantes dans les rapports d'enquête de l'entre-deux-guerres au seuil des années 50 .

12. SHGN, 14.999 (Maine-et-Loire), BT Maulévrier, 4/4, 4 septembre 1944.

13. À titre d'exemples : «Son union avec le garde républicain P. ne peut nuire à la considération de l'Arme ", SHGN, 52 E 79 , BT Chaumont, 96/4, rapport du 19 novembre 1938 ; « Mademoiselle E, réputée économe et travailleuse, est digne de l'union projetée », SHGN, 52 E 79, BT Bourbonne-les-Bains, 13/4, rapport du 29 juillet 1942.

14. Boyé $1975: 6,10$.

15. Instruction $n^{\circ} 011040 \mathrm{DN} / \mathrm{Cab} / \mathrm{EMP}$ du 30 mars 1957 fixant les conditions dans lesquelles les militaires de la gendarmerie nationale peuvent être autorisés à contracter mariage. SHGN, Mémorial de la gendarmerie, 18ad.79, p.55-65.

16. Circulaire $n^{\circ} 31150$ Def/Gend/Emp/Serv du 19 juin 1978 relative au mariage des officiers et sous-officiers de gendarmerie. SHGN, Mémorial de la gendarmerie, version CDRom et intranet pour les années les plus récentes.

17. Débats parlementaires du Sénat, séance du 2 juin 1972, discussion de l'article 13 du projet de loi sur le statut des militaires : rejet d'un amendement communiste visant à étendre aux gendarmes la dispense d'autorisation préalable de mariage.

18. Boyé $1975: 6$.

19. Lambert $1984: 53$.

20. Des femmes de gendarmes apparaissent à l'origine de certaines lettres ou pétitions : Dieu 1999. De même, elles sont nombreuses à s'exprimer dans la presse à l'occasion de cette crise.

21. Constitué au printemps 2000 en groupe de pression quasi para-syndical, le mouvement des femmes de gendarmes a été un relais important de la contestation qui a éclaté fin 2001. Dieu $2002: 84$.

22. Image retenue par Daniel Schneidermann dans les colonnes du quotidien Le Monde, 18 août 1989, p. 7.

23. Signalons au passage le principe des visites dites « inopinées » (en fait l'intéressé est informé $48 \mathrm{~h}$ à l'avance) des appartements privés par la hiérarchie, afin de vérifier l'état du casernement. De même, une déclaration est-elle nécessaire pour tout hébergement temporaire d'une personne extérieure à la famille.

24. Témoignage d'une épouse de gendarme, Libération, 18 août 1989, p. 4.

25. Témoignage dans France Dimanche, 9 septembre 1989, cité dans Dieu 1999 : 417. Sans doute l'exemple peut-il apparaître anecdotique, mais il n'en demeure pas moins significatif. F. Dieu cite également un dossier du magazine Maxi, « Témoignage bouleversant d'une femme de gendarme », 3 octobre 1989.

26. Boyé $1975: 10$.

27. «La femme de gendarme a le sentiment d'être un point de mire », « On vous observe, on vous juge, on ne vous pardonne rien », extraits d'entretiens, Boyé 1975 : 6 .

28. La formation des gendarmes insiste beaucoup sur la vie de famille, comme vecteur d'équilibre et de soutien, indispensable à la réussite et à l'efficacité professionnelles.

29. Reynaud $1988: 129$.

30. Récente (1972) et longtemps ralentie par des quotas de recrutement (levés en 1998), la féminisation de la gendarmerie reste limitée et inégalitaire. Avec 3577 femmes sur un effectif total de 96820 en 2001 (soit 3,7\%), la gendarmerie dispose du taux de féminisation le plus faible des trois armes (Terre, 7,45\% ; Air, 10,66\% ; Mer $7,34 \%$ ). Elles restent, par ailleurs, encore souvent cantonnées dans des fonctions subalternes : 0,5\% de femmes officiers seulement. Dieu $2002: 140$. 
31. Dieu 2002 : 142. Lors du mouvement de 1989, certaines pétitions s'étaient déjà émues de cette situation, ainsi celle des gendarmes de l'Oise : « Pensez-vous que les 30\% de démissions du personnel féminin dans les trois premiers mois d'activité soient logiques ? », Le Monde, 6-7 juillet 1989, p. 14.

32. Reynaud $1988: 129$.

33. Dieu 2002 : 209.

34. Manuel de « savoir-vivre » à destination du personnel, il définit des codes, normes et règles à suivre au quotidien.

35. Le titre complet du volume cité est : Le guide du gendarme dans certaines circonstances de sa vie privée et de sa vie professionnelle, SHGN, sans date, p. 94.

36. Dieu $2002: 245-246$.

37. Dieu 2002 : 208.

38. C'est l'une des motivations du mouvement de 1989. Sous le titre « Le blues d'une caserne ordinaire », le quotidien Libération présente une brigade territoriale du Languedoc au sein de laquelle « en quelques mois, sur huit familles, trois femmes de gendarmes ont demandé le divorce et ont déserté les lieux », 18 août 1989, p. 2. La lettre ouverte de gendarmes de l'Oise à Jean-Pierre Chevènement interpelle le ministre de la Défense sur ce thème : « Pensez-vous qu'il soit normal que le nombre de divorces parmi la population gendarmique soit élevé ? ", Le Monde, 6-7 juillet 1989, p. 14. François Dieu signale l'augmentation des situations de célibat géographique et s'interroge sur son impact en terme de divorce, tout en reconnaissant que le phénomène est difficilement quantifiable. Dieu 2002 : 257.

\section{RÉSUMÉS}

Appuyé sur les textes normatifs, relatifs au mariage et à la réglementation de la vie privée des gendarmes, cet article témoigne du contrôle matrimonial mais aussi social imposé aux femmes de gendarmes en raison du statut professionnel de leur mari. En effet, la dimension totale de la fonction gendarmique tend souvent à effacer ou gommer la séparation entre vie et espace professionnels et privés. Chemin faisant, les épouses sont, bon gré mal gré, partie intégrante de la communauté professionnelle de leur mari. Pendant une grande partie du siècle, ce mode d'organisation a été profondément enraciné dans la culture du corps, participant de son identité. Nul doute cependant, que les contestations internes de 1989 et 2001, mais aussi la féminisation récente de la gendarmerie, conduisent aujourd'hui à des évolutions dans la façon d'appréhender les rapports de genre au sein de l'institution.

This article uses normative texts concerning constables' marriage and private life in order to illustrate both the marital and social control that was imposed on their wives due to their professional status. The all-encompassing quality of a constable's profession tended to erase or to blur the normal separation existing between the lives and spaces of professional and private life. As a result, wives, whether they wanted it or not, became integral elements of the professional communities of their husbands. Throughout much of the century, this organizational mode was profoundly anchored in the professional culture of this group and contributed to its identity. Unquestionably, however, internal protests in 1989 and 2001, as well as the recent feminization 
of the constabulary, have introduced changes in the ways gender relations are negotiated within the institution.

\section{INDEX}

Mots-clés : caserne, femmes, mariage, gendarmerie

Index géographique : France

\section{AUTEUR}

\section{MARC BERGÈRE}

Marc Bergère est maître de conférences d'histoire contemporaine à l'Université Rennes 2, membre du Centre de Recherches Historiques sur les Sociétés et Cultures de l'Ouest (CRHISCO/ CNRS) et chercheur associé au Service Historique de la Gendarmerie Nationale dans le cadre de recherches relatives à l'épuration au sein de la Gendarmerie à la Libération. Travaillant sur l'histoire sociale et culturelle de l'épuration, Une société en épuration. Le Maine-et-Loire de la Libération au début des années 50, Rennes, PUR, 2004, il a également publié « Différence des sexes et répression judiciaire pour faits de collaboration : approche comparée des deux guerres mondiales ", dans Capdevila Luc, Cocaud Martine, Godineau Dominique, Rouquet François, Sainclivier Jacqueline (dir.), Le genre face aux mutations. Masculin et féminin, du Moyen-Âge à nos jours, Rennes, PUR, 2003, pp. 327-336. 